\title{
I mpact and sustainability of an accredited paediatric nursing training programme in Ghana
}

\author{
Bonnie Stevens ${ }^{1}$, Ernestina Safoa Donkor ${ }^{2}$, Bamenla Quam Goka ${ }^{3}$, I saac Odame ${ }^{1}$, Margrieta Langins ${ }^{1}$, \\ Shirine Riahi ${ }^{1}$, Stephanie de Young ${ }^{1}$, Dylan Walters ${ }^{1}$, Celia Eliason ${ }^{2}$, Bonnie Fleming-Carroll ${ }^{1}$, Grace \\ Barnes $^{4}$, Francis Victor Ekey ${ }^{5}$, Pam Hubley ${ }^{1}$, Felix Nyanteh ${ }^{6}$, Eva Mensah ${ }^{4}$, Ivy Sackey ${ }^{4}$ \\ 1. The Hospital for Sick Children, Toronto, ON, Canada. 2. School of Nursing, College of Health Sciences, University of \\ Ghana, Legon Boundary, Accra, Ghana. 3. University of Ghana Medical School, Korle-Bu, Accra, Ghana. 4. Ministry of \\ Health, Ministries, Accra, Ghana. 5. Ghana Health Service, Human Resource Directorate, PMB, Ministries, Accra, Ghana. \\ 6. Nursing and Midwifery Council of Ghana, Accra, Ghana.
}

Correspondence: Bonnie Stevens. Address: The Hospital for Sick Children, 555 University Ave., M5G 1X8 Toronto, ON, Canada. Email: bonnie.stevens@sickkids.ca

Received: September 8, 2014

DOI : $10.5430 /$ jnep.v4n12p142
Accepted: October 8, 2014

URL: http://dx.doi.org/10.5430/jnep.v4n12p142

\section{Abstract}

Objective: In this qualitative descriptive study, we explored the perceived impact and sustainability of the first accredited Paediatric Nursing Training Programme (PNTP) in Ghana, established in 2010 by a north-south Ghanaian-Canadian partnership to address child health care access and quality issues in the country.

Methods: A fundamental qualitative descriptive approach was used. Focus groups and individual interviews were conducted with 44 stakeholders including graduates, their clinical associates, PNTP faculty, and key health system administrators in Accra and Tamale in November 2013. Interview questions were guided by a Performance Measurement Framework (PMF) that was developed in collaboration with partners to evaluate progress towards meeting the programme objectives. Whole group content analysis was conducted to achieve an overall collective impression of the PNTP from all of the stakeholders.

Results: Four themes emerged from the data which offered insight into programme successes and current challenges from a local perspective: a) addressing system gaps through specialized nursing training, b) strengthening nursing identity, c) building nursing competencies and practices, and d) inspiring innovative pedagogy in nursing education.

Conclusions: PNTP strengths are well aligned with modern systems-based approaches to health professional education; by maintaining this focus with future design recommendations, the PNTP will continue to serve as an international exemplar of health human resource planning and education for equity in health.

\section{Key words}

Education, Ghana, Health resources, Nursing, Nursing education research, Paediatric nursing, Programme evaluation

\section{I ntroduction}

\subsection{Enhancing access to paediatric nursing care in Ghana}

Child mortality in developing countries remains unacceptably high. Although the under-five mortality rate in Ghana has decreased from 122 to 72 deaths per 1,000 live births between 1990 and $2012^{[1]}$ and is trending in the right direction, 
treatable illnesses such as malaria, respiratory infection, diarrheal disease, sickle cell disease and neonatal causes of death continue to claim young lives. Challenges to child survival interventions in Ghana can in part be attributed to understaffing in health facilities, inequitable distribution of health resources between urban and rural/remote areas, de-motivated staff, and health workers with inadequate skills for effective health care delivery ${ }^{[2-7]}$.

In 2009, the Ministry of Health (MOH) in Ghana committed to training 1500 specialty paediatric nurses, within 10-15 years, to improve child survival. Often, nurses are the only health professionals available in rural and remote regions in Ghana ${ }^{[4,8]}$. At the time, there was no paediatric nursing training programme or licensing exam in the country, and there were only six nurses trained in the paediatric specialty as a result of training abroad. The MOH, Ghana Health Service (GHS), Korle Bu Teaching Hospital (KBTH), Nursing and Midwifery Council of Ghana (N\&MC), and University of Ghana School of Nursing (UG-SON) partnered with The Hospital for Sick Children (SickKids) in Toronto, Canada, to: a) develop the content and infrastructure for Ghana's first accredited paediatric nursing curriculum and b) prepare an initial cadre of 150 paediatric nurses. A collaborative approach in developing, implementing, and evaluating the curriculum was taken to ensure its relevance in the local context and sustainability beyond the project period. This initiative was made possible with financial support from the Government of Canada, provided through the Department of Foreign Affairs, Trade, and Development (DFATD), and the SickKids Foundation.

\subsection{The Ghana-SickKids paediatric nursing training programme}

The Ghana-SickKids Paediatric Nursing Training Programme (PNTP) was designed as an intensive one-year programme to equip nurses with essential clinical skills, knowledge, and professional abilities in child health to prepare them to be leaders and mentors in their field. The curriculum design ensures the integration of theory with practice and active engagement of the learner by incorporating a combination of didactic teaching, interactive case-based learning, simulation activities, reflective journaling, and clinical coaching. Core content includes family-centered care; stages of child growth and development; anticipatory guidance for families and care providers at each stage of a child's development; cultural context and gender equity; assessing, planning, and evaluating nursing interventions related to changes in a child's health condition; timely and appropriate response to health care emergencies; and enhancing nursing professionalism and leadership within the inter-professional health care team. Graduates are awarded a post-basic certificate by the UG-SON and are eligible to write the newly developed N\&MC licensing exam for paediatric nurse specialists. Between 2011 and 2014, an initial cadre of 222 paediatric nurses was trained in the first 4 cohorts, including programme graduates $(n=153)$ and those currently in training $(\mathrm{n}=69)$.

\subsection{Evaluating the PNTP}

The purpose of this study was to explore key stakeholders perceptions of the impact and sustainability of the PNTP in Ghana after three years of offering the programme. The evaluation was guided by a Performance Measurement Framework (PMF) that was developed in collaboration with partners to evaluate progress towards meeting the programme objectives including, in the short-term: a) the acquisition and practice of paediatric nursing knowledge, skills, and leadership abilities within the health system in Ghana, and b) ensuring PNTP relevance to sustain and potentially expand the programme.

\section{Methods}

\subsection{Ethical approvals}

Prior to the start of the study, ethical approvals were obtained from GHS Ethical Review Committee, the Noguchi Memorial Institute for Medical Research Institutional Review Board at the University of Ghana, and the Research Ethics Board at SickKids hospital. Institutional and ethical requirements for transferring and storing data were followed as per the data sharing agreement. 


\subsection{Design, sample, and setting}

A fundamental qualitative descriptive approach ${ }^{[9]}$ was used. We selected this approach for the emphasis on descriptive and interpretive validity, so we could find comprehensive and straight (minimally theorized) answers to programme evaluation questions on the impact and sustainability of the PNTP in Ghana. We purposefully interviewed four categories of stakeholders to maximize the variation of perspectives on the programme; our inclusion criteria were: a) graduates from the first two cohorts of the PNTP, who subsequently passed the N\&MC licensing examination, and worked in either a clinical or education setting, b) faculty from the UG-SON who were students in the first cohort of graduates and who subsequently taught the PNTP curriculum, c) clinical informants (i.e., paediatricians, head nurses and Nursing Officers) who worked with the licensed paediatric nurses in hospitals or in the community, and d) health system informants who provided input, assistance, oversight or authority on the development, financing, regulation, or implementation of the PNTP. The study was conducted in November 2013 in two major Ghanaian cities: Accra, the capital and largest city of Ghana, and Tamale, the Northern Region Capital. The majority of licensed graduates from the first and second cohorts were working in these regions, and the PNTP was delivered at the UG-SON in the Greater Accra Region (GAR). In the GAR the under-five mortality rate is below the national child mortality average, and in the Northern Region it is above the national average ${ }^{[10]}$.

\subsection{Procedures and measures}

\subsubsection{I nterview guide}

A semi-structured interview guide that was based on the PMF and (short-term) programme objectives was used to conduct focus group and individual interviews. Questions and probes were designed to encourage participants to share their experiences, opinions, and insights in relation to the PNTP. The interviews began more generally to garner participants' overall views about the paediatric nursing training, and then proceeded to more specific queries about: a) changes in graduates' clinical practices, b) their influence in the workplace, c) factors affecting their application of acquired skills, knowledge, and leadership abilities, and d) requirements to sustain and develop the programme to meet health system, individual patient, and population needs. All interviews were conducted in English.

\subsubsection{Focus groups}

Three focus groups were conducted with five to eight licensed graduates in each group. The focus group format was used to enable the sharing and comparing of individual experiences, insights and ideas, and contributed to the overall depth and richness of conversation. Thirty-six graduates met the inclusion criteria and were notified of the study through a mailed letter and follow-up text message that were sent after an introductory letter from the university. The letters explained the purpose of the study, data collection methods, and time commitment. The first 16 respondents in the Greater Accra Region and the first six respondents in the Northern Region were invited to participate, reflecting enrollment from these regions in the first two cohorts of the programme. Two of the focus groups took place at a teaching hospital in Accra, and one at a teaching hospital in Tamale. Each focus group lasted 60 to 90 minutes and was conducted in a private room designated for the study by the hospital administration. Participants were compensated for their travel costs and provided with a small honorarium (5.00 Ghana cedis phone card) in appreciation of time taken for their participation.

The focus groups were facilitated by two experienced interviewers - one Canadian senior nurse researcher (BS), and one nursing faculty member from Ghana (CE) - who were not involved in the delivery of the PNTP. Both interviewers were members of the PNTP evaluation committee that developed the PMF. On the day of the focus group, participants were provided with a detailed explanation of the study, confidentiality requirements, and procedures for anonymity in accordance with privacy legislation. Written consent for participation and audio-taping was obtained from each participant. All focus groups were audio-taped and professionally transcribed verbatim. Any identifying information was removed during transcription. 


\subsubsection{I ndividual interviews}

Individual interviews facilitated the expression of personal experiences, insights, and ideas from faculty, clinical informants, and system informants that was not influenced by the views of others. The participants were recruited in the same manner as the PNTP graduates, with a total of 24 invitations sent. Of those invited, 20 were able to participate. Individual interviews were arranged in a private space at the workplace for each informant, in either Accra or Tamale, and each interview lasted 30 to 45 minutes. The interview structure, consent, and data collection processes were similar to the focus groups with graduates. The same interviewers were involved, in addition to one Canadian nurse research coordinator (ML).

\subsection{Data Analysis}

Following completion of all interviews and transcription, three research coordinators (SR, ML, and JB) with coding experience reviewed the transcripts using whole group content analysis ${ }^{[11]}$ to achieve an overall collective impression from all of the PNTP stakeholders. This approach included development of the initial coding framework, establishing reliability among coders, and coding all data until no new categories emerged. Categories and subcategories were continually generated, redefined, explored and collapsed during the coding process, and overarching themes and subthemes were generated. Data analysis was conducted manually using Microsoft Office 2007 Excel Worksheets.

\section{Results}

A total of 44 participants were recruited into the study: 20 licensed PNTP graduates, 4 faculty, 10 clinical informants, and 10 health system informants. Participant demographics are detailed in Table 1. Overall, feedback about the PNTP was positive. Four overarching themes emerged from the data capturing perspectives on the impact and sustainability of the programme: a) addressing system gaps through specialized nursing training, b) strengthening nursing identity, c) building nursing competencies and practices, and d) inspiring innovative nursing pedagogy. Selected quotations illustrate the themes described below.

\subsection{Addressing system gaps through specialized nursing training}

\subsubsection{I mpact}

Participants were very mindful that the paediatric nursing training programme was addressing critical gaps in the standard of nursing education in Ghana. Up until the introduction of this programme:

Paediatric nursing has been neglected as a specialty in this country. We haven't had any specialty training in paediatric nursing. (system informant)

Participants were optimistic this training could off-set gaps in population access to quality paediatric care services once a critical mass of nurses was trained:

By the time we build that solid base, I think we'll really see the impact. It's the beginning and I really think it'll make a difference. (paediatrician)

\subsubsection{Sustainability}

Whether the PNTP will succeed in fully addressing gaps in nursing education and access to paediatric care will depend on: a) expanding programme enrollment and delivery sites, b) emphasizing graduate deployment in the districts and primary care, c) appealing to new recruits, and d) ensuring ongoing programme support. Recommendations were made to expand programme enrollment to include community health nurses and midwives, to improve access to care for children in remote areas not close to hospital centres. Graduates from the Northern part of Ghana thought the programme ought to be delivered at a university in their region to further enhance access to paediatric nursing education and care; the implicit assumption being that nurses will continue to work in the area after graduation. Several graduates suggested there should Published by Sciedu Press 
be work incentives for deployment in the remote regions. The majority of study participants agreed that communities outside the major city centres and institutions other than hospitals needed greater access to paediatric primary nursing care.

Once these things [delivery of paediatric nursing care] are being done I believe it will help. But where it is only centered in the hospital they are only waiting for somebody to fall sick and come back and then you give the care. I think we keep on going around in circles and where you will get to them are the districts and the schools, at the mosques, at the church; I believe that will make an impact. (system informant)

Table 1. Study Participant Demographics

\begin{tabular}{|c|c|}
\hline Participant & Demographics \\
\hline \multicolumn{2}{|l|}{ PNTP licensed graduates $(n=20)$} \\
\hline \multirow{2}{*}{ Gender } & $90 \%$ women $(n=18)$ \\
\hline & $10 \%$ men $(n=2)$ \\
\hline \multirow{2}{*}{ PNTP Cohort } & $65 \%$ first cohort $(n=13)$ \\
\hline & $35 \%$ second cohort $(n=7)$ \\
\hline \multirow{2}{*}{ Employment Region } & $75 \%$ GAR $(n=15)$ \\
\hline & $25 \%$ Northern Region $(n=5)$ \\
\hline \multirow{3}{*}{ Employment Type } & $60 \%$ teaching hospitals $(n=12)$ \\
\hline & $25 \%$ district hospitals $(n=5)$ \\
\hline & $15 \%$ polyclinics, mission, and quasi-government health facilities $(n=3)$ \\
\hline \multirow{5}{*}{ Highest Level of Education Reported } & $50 \%$ nursing diploma $(\mathrm{n}=10)$ \\
\hline & $10 \% 0$ level (secondary school) certificate $(n=2)$ \\
\hline & $10 \%$ nursing certificate $(\mathrm{n}=2)$ \\
\hline & 25\% university-based nursing degree $(n=5)$ \\
\hline & $5 \%$ not specified $(n=1)$ \\
\hline \multicolumn{2}{|l|}{ Faculty $(n=4)$} \\
\hline \multirow{3}{*}{ Employment Status at University } & 1 full-time lecturer \\
\hline & 1 part-time lecturer \\
\hline & 2 assistant lecturers \\
\hline Highest Level of Education Reported & $100 \%$ Master’s prepared \\
\hline \multicolumn{2}{|l|}{ Clinical informants $(\mathbf{n}=10)$} \\
\hline \multirow{2}{*}{ Employment Region } & $80 \%$ GAR $(n=8)$ \\
\hline & $20 \%$ Northern Region $(n=2)$ \\
\hline \multirow{3}{*}{ Professional Category } & 4 paediatricians \\
\hline & 5 head nurses \\
\hline & 1 nursing officer \\
\hline \multicolumn{2}{|l|}{ System informants $(n=10)$} \\
\hline Region & $100 \%$ GAR $(n=10)$ \\
\hline \multirow{4}{*}{ Health System Representation } & 2 members from N\&MC \\
\hline & 4 executive-level nurses from GHS and $\mathrm{MOH}$ \\
\hline & 3 hospital nursing administrators \\
\hline & 1 member of the College of Health Sciences at UG \\
\hline
\end{tabular}

The awarding of a PNTP certificate at the academic level was perceived by many as a deterrent to new students enrolling in the programme, as degrees and diplomas have greater status and are associated with a higher compensation scale. This sentiment persisted despite the GHS giving approval for licensed graduates to be eligible for increased compensation equivalent to post-basic diploma training and in alignment with all other post-basic specialist training in Ghana. Graduates, faculty, and clinical informants applauded existing support from the hospitals to subsidize students and emphasized that this support remain a priority. At the same time, participants called for even greater system-wide support for the 
programme through increased awareness of the programme and its merits, and sustained priority funding to be able to continue and expand the PNTP:

It needs commitment of management at the highest level to make it something that will last, that will be part of the fabric of nurse training in Ghana, that will not suffer if the demands are too many on the budget or the system, and it must have a line. A budget line. Both within the university and in the ministry and also the service areas like the teaching hospitals that are autonomous. (system informant)

\subsection{Building nursing competencies and practices}

\subsubsection{I mpact}

Paediatric nurses were thought to have improved competencies as a result of their training in the PNTP, which were reflected in new activities or practices by the nurses. A substantive list of diverse competencies was identified that aligned with the PNTP curriculum and fell into one of four categories: a) clinical skills and knowledge, b) leadership and decision making, c) inter-professional collaboration, and d) family-centered care. The nurses demonstrated the clinical skills and knowledge to manage patients and improve the overall efficiency and flow of care. As one paediatrician noted,

Now you are working with nurses who know what to do in an emergency even before they call you; their assessment of the child's situation is almost like what you would assess yourself . . . it makes things easier because you're not starting from scratch all the time. They have information for you by the time you get to a particular patient so it helps to channel your efforts better. They intervene and ask for help when needed.

The nurses were also shaping health care delivery and culture through their leadership activities and inter-professional and family-centred approaches to care. Examples of their leadership activities included checking for medication errors, voicing clinical concerns to physicians, initiating requests for paediatric-appropriate resources to supply their units, teaching in the community, and taking on charge nurse activities when necessary, such as assigning tasks to other team members and consulting on complex cases.

Nurses were also contributing routinely and with more confidence in team discussions, presenting at departmental rounds, and participating in staff development sessions. The nurses introduced a culture of care driven by family and child needs. In some cases nurses formally changed roles or departments as a result of their paediatric training, but in many cases the aforementioned activities or practices occurred within existing roles.

\subsubsection{Sustainability}

The ability to apply and sustain new competencies and practices will depend on how role confusion is addressed in the workplace. Without a defined paediatric nursing scope of practice and clear job descriptions, nurses worried about professional boundaries:

... because sometimes you feel you are doing something overboard or something under. So when you know your job description, then you know you are working within your limits. (paediatric nurse)

Lack of a clear role description also made it difficult to monitor the nurses' performance for quality assurance and professional development purposes:

... we should have guidelines, policies, that will state that paediatric nurses who work in this particular area, this is what they are supposed to do, this is their scope of practice. These things are not available. So in monitoring or supervising them, how do we measure best practices? (system informant)

In addition, the paediatric nursing certificate did not guarantee the kind of recognition or career advancement nurses could expect for their new skill set. For nurses who were employed in senior positions: 
Because you have a higher level before this training, they [administrators] feel that you don’t qualify [for a promotion]. (paediatric nurse)

No consensus emerged on whose responsibility it was to articulate the paediatric nursing scope of practice and whether this should be the role of the graduates themselves through a self-regulated professional association, the employers who provide the services, or the accreditation body, the N\&MC, who approved the curriculum.

Similar to Ghanaian physicians training in the West, there were concerns about the paediatric nurses being able to practice their new competencies without medical resources (e.g., equipment, supplies, and medication):

If we are able to get the needed equipment because at times we may have the know-how, we are willing to do the work but because of the lack of resources we can’t perform certain things. (clinical informant)

A nurse from the Northern Region suggested coordinating with some national non-governmental organizations to:

... help out in areas where there are shortfalls in logistics ... especially in equipment to manage these babies and children ... especially in the Northern part; I think we have so, so much lacking. We don't have a lot of the things to work with. We are only improvising. (paediatric nurse)

\subsection{Strengthening nursing identity}

\subsubsection{I mpact}

A stronger nursing identity appeared to be developing among the paediatric nurses, grounded in their new competencies. Part of this new identity included a change in the nurses' feelings and attitudes towards their work.

I get the impression that there's more meaning to their nursing work now; it's not nursing as always but it's nursing with a goal. That's the impression I get. They seem a lot more focused now than they were in the past with just doing their regular nursing. (paediatrician)

They expressed feelings of empowerment, confidence, job satisfaction, and enthusiasm:

I think that we've been empowered by so much knowledge and new skill because prior to entering the programme, irrespective that we'd been working in the department for so many years, there were some areas that we were not exposed to. But now I can say personally that I can confidently nurse. (paediatric nurse)

I feel satisfied. I have job satisfaction now. (faculty)

They came [out of the programme] with a lot of venom - they came with a lot of enthusiasm. (paediatrician)

Taking on new nursing activities or practices was enhancing the nurses' image as skilled professionals, and the nurses were increasingly being perceived as valuable contributors, role models, and inspirations by their colleagues.

\subsubsection{Sustainability}

Graduates were concerned about professional isolation and lack of quality assurance processes following programme completion diminishing their momentum or enthusiasm, and ability to stay current in their practices:

We have started with the zeal, but there is no continuous monitoring. After training nobody monitors. It ends there ... a after training there is nothing like somebody coming to monitor what you are doing or give you that kind of improvement opportunity. (paediatric nurse)

In the immediate term, some graduates were turning to social media sites like Facebook for clinical support. Others suggested a formal paediatric nursing association with regular meetings might be effective to meet their needs: 
We don't have one meeting within a quarter or yearly to actually deliberate on issues concerning paediatric nursing care. All we have is everybody is in their place working. (paediatric nurse)

\subsection{I nspiring innovative pedagogy in nursing education}

\subsubsection{I mpact}

Nurses who completed the PNTP described the curriculum as inspiring a new pedagogy in nursing education based on respect for students, adult learning principles, and interactive and problem-based learning versus didactic teaching methods alone. This approach to teaching and learning helped hone their skills in communication, patient history taking, physical assessments, paediatric advanced life support/resuscitation, and pain assessment and management, for example. The graduates valued leadership development and the opportunity to shape their learning experience:

The programme was very interesting because it was like a discussion. You bring your own ideas. It's not like somebody teaching you what to do, or what to learn. But it was something you bring out of yourself. So you develop self a lot through the programme because it’s more participatory. (faculty)

This more humanistic approach to education influenced how the students who completed the programme planned to teach as faculty in the programme, or educate families in the clinical setting, by drawing on core content in family centred care, social determinants of health theory, and health belief model theory.

\subsubsection{Sustainability}

Concerns about the sustainability of the university programme were focused on: a) faculty capacity to meet teaching demands, b) infrastructure challenges (i.e., space), and c) curriculum expansion (i.e., demands for continuing education, distance education, upgrades to a post-basic diploma or degree, and development of a Master's degree offering). Faculty discussed teaching loads and pressure for them to upgrade their credentials to the doctorate level:

Time is a problem because I'm teaching our regular students and at the same time teaching the PNTP students.... It's a bit difficult for me in terms of sitting down to prepare my notes for my regular students, and marking the scripts, because I have a class of about 150 .

Faculty and graduates identified infrastructure challenges associated with a general lack of protected space for the programme at the university. For example, faculty requested a dedicated library for the PNTP:

Well the place is very small. We need a library [for the programme]. Up until now we have not put our books in a place where somebody can just go in and sit down and read.

The overarching desire is for the programme to be expanded into more regions, either through distance education or satellite training sites. There were requests for continuing education offerings in paediatrics (e.g., workshops) for graduates and other nurses. In terms of the curriculum content: a) for some, the content was considered too specialized for a certificate programme and they wanted the certificate upgraded to a post-basic diploma or degree; b) for others, the curriculum was too condensed or rushed, and they suggested developing a two year long Master's degree with additional content in pharmacology, physiology, emergency medicine, and policy development; c) for others, the one year certificate was satisfactory although they requested more supervised clinical learning experiences and mentoring from paediatricians and nursing leaders.

\section{Discussion}

A systems approach was used to interpret the research results, based on the Integrative Framework of Health and Education by the Global Independent Commission on Education for Health Professionals for the $21^{\text {st }}$ Century ${ }^{[12]}$, referred to as the Commission in this paper, in conjunction with human resources for health literature ${ }^{[4,6,7,13-18]}$. These resources 
were helpful in understanding the PNTP strengths and areas for future development in promoting greater health equity through health professional education.

\subsection{Criteria for admission}

According to the Commission, criteria for admission to health professional programmes should reflect population needs and health equity principles through balanced rural, ethnic, gender, and socio-cultural recruitment policies; not just academic-based admission criteria alone ${ }^{[12]}$. Graduates from the first two PNTP cohorts were successfully recruited from all 10 regions in Ghana following a nomination and interview process; however, the majority was from the GAR to build the teaching infrastructure (i.e., clinical preceptors and faculty) in the region where the programme was delivered. Leaders from the UG-SON chose faculty to complete and then teach the programme. Local hospital authorities from each region nominated a predetermined number of candidates based on their clinical experience, interest, and leadership capacity who they anticipated would become clinical preceptors for future students. Most stakeholders agreed programme enrollment should be increased and recruitment extended more widely in subsequent cohorts, to increase access to paediatric care in all communities and health facility types.

\subsection{Competencies}

Patient-centred and needs based competencies to address individual and population health are also hallmarks of the Commission framework ${ }^{[12]}$. The PNTP was overwhelmingly perceived as a mechanism for enhancing nurses' paediatric competencies, including their capacity to lead, communicate effectively, and incorporate inter-professional and family-centered care principles. The graduates' abilities to involve patients using family-centered care principles and their increased concern and desire to advocate for patients within the interdisciplinary team is a starting place to shape a culture of care that is driven by individual or population needs.

The explicit articulation of paediatric nursing competencies for employers and other stakeholders to better utilize the graduates' knowledge and skills to optimize patient care in the workplace was lacking, leading to role confusion. Addressing this problem would contribute to much needed role clarity in the health system, overall. The Commission ${ }^{[12]}$ proposes that applying standardized discipline-based competencies in addition to core health care competencies (e.g., in family centred care and inter-professional team-based practice) would enable distinguishing the different health professions, aligning roles with compensation, and challenging credential creep whereby ever greater credentials are required for a specific position.

\subsection{Teaching methods}

The PNTP used evidence-based teaching methods not often employed in developing countries, but known to be effective in those settings ${ }^{[13-15]}$. The graduates, including faculty, thought these novel teaching methods enhanced their learning. Their emphasis on the role the skills lab and training in the clinical practice setting had on enhancing classroom learning and developing the nurses' clinical skills highlights the importance of hands-on active learning, in the local population context. Although feedback was positive, the challenge will be developing local faculty to continue using these interactive methods to teaching and learning.

Graduates from the northern and more remote regions, where diversification of learning channels becomes more important, identified the internet and social media as modalities they have used to access information and support; to connect with peers for case-by-case and overall support. As the use of information technology expands in health care, the need for a corresponding shift in competencies in information management - finding, synthesizing, analyzing, and applying information in practice for evidence-based decision making — increases ${ }^{[12]}$. The programme will need to evolve and equip students with skills to utilize information technology to provide evidence-based care. 


\subsection{Career pathways}

Education has a key role to play in influencing career pathways students chose to take - e.g., following traditional urban biases to work in hospitals or servicing more remote and disadvantaged populations - by shaping their professional identity alongside their clinical knowledge and skills development ${ }^{[12]}$. As part of the leadership content of the PNTP curriculum, each student designed and implemented a service improvement project (change project) to address an immediate child health issue. This component of the curriculum enhanced confidence, collaboration, communication, and leadership ability and how it translates into real change. Choice of work location (rural or urban) will most likely be influenced by where graduates live, undertake their training, financial incentives, compulsory service programmes, and the availability of professional and personal support which includes professional development opportunities and support networks ${ }^{[4,6,7]}$.

\subsection{Regulation considerations: local, national, and global}

Health education systems should be regulated with a local, national, and global view to promoting joint planning, knowledge sharing, standard setting, and a culture of critical inquiry to improve equity in health ${ }^{[12]}$. The country has been successful in placing the capacity development of Human Resources for Health (HRH) at the center of its health policy ${ }^{[7,16-18]}$, and a HRH Strategic Plan 2007-2011 which clearly prioritizes the strengthening and development of an appropriately skilled, motivated, and equitably distributed health workforce, including the nursing workforce ${ }^{[14]}$, has been developed. Roles of the different health professional regulatory bodies and educational institutions are very clear. For example, the MOH has delegated the N\&MC with the regulation of nurses and midwives, including the PNTP graduates. The UG-SON or $\mathrm{MOH}$ training colleges are accountable for delivering nursing programmes based on a set curriculum worked out in collaboration with the N\&MC and the GHS (the executive body of the MOH). Certificates, diplomas, and degrees are awarded by the educational institutions, while professional licensing exams are administered by the N\&MC. The PNTP worked within this existing infrastructure.

The accreditation criteria for the PNTP curriculum may need to be reviewed on a regular basis to ensure programme goals are met and the quality of the student learning experience. It will be important for those involved in this process to integrate local stakeholder feedback on the programme and evaluate any changes. The pressing concern from stakeholders around the final graduation designation (certificate versus diploma or degree) will need to be re-examined. Degree-level training would conceivably limit programme accessibility due to the increased duration of education and admission criteria ${ }^{[19]}$, which is contrary to the PNTP goal to increase access to paediatric nursing care. These issues will need to be resolved and may involve rethinking work incentives, not only accreditation.

Other regulatory concerns that will require attention include plans for programme expansion, developing faculty to teach the programme, defining paediatric nursing scopes of practice, and securing programme finances. In terms of finances, up to $90 \%$ of the health budget goes to salaries ${ }^{[17,18]}$, placing constraints on the funds available for service delivery and support of staff development in Ghana. Different financing arrangements and sources will need to be secured to sustain, revise, or develop the innovations introduced through the PNTP. The ability to attract additional and more sustainable programme financing will serve as an important gauge for wider system support for the programme and nursing in advancing child health in Ghana.

The north-south partnership underlying the PNTP has begun to harmonize paediatric nursing education in Ghana with North American standards, while adapting to the local context. However, care must be taken to ensure the partnership does not become too heavily dependent on one side, as is the challenge for many international partnerships ${ }^{[20,21]}$. To ensure more ownership of the programme, the UG-SON faculty has taken over training the third and fourth programme cohorts. New global connections, such as faculty exchanges and global research collaboratives, should be fostered to build capacities on both sides and ensure currency in education and research for improved population health. 


\subsection{Study Limitations}

The sample of graduates was limited to those who passed the licensing exam and were from the GAR and Northern Regions. The data collection methodology was based on the assumption that perceptions about the programme were willingly shared, accurate, and not subject to reporting bias. Further study on the long-term impact of the PNTP on clinical, educational, and population outcomes, using additional validated measures, is necessary.

\section{Conclusions and recommendations}

Overall, the PNTP has achieved a reputation among key nursing education and health system stakeholders in Ghana for addressing a gap in nursing education and population access to paediatric health care. This programme is perceived by a wide variety of stakeholders to have fostered a positive and expanded nursing identity in Ghana, and enhanced nurses' clinical competencies, including their capacity to lead and incorporate inter-professional and family-centered care principles. The PNTP has inspired the next level of pedagogy in nursing education in Ghana that goes beyond rote learning to enhance student engagement and learning through evidence-based experiential and situational learning. Graduates are employing new practices to improve care delivery in hospitals, communities, and districts. PNTP strengths are well aligned with modern systems-based approaches to health professional education. Despite these successes, ongoing sustainability of the programme is a key challenge. The PNTP evaluation committee has outlined five priority recommendations, based on the results of this study (see Table 2). By maintaining a systems approach with these recommendations, the PNTP will continue to serve as an international exemplar of modern health human resource planning and education to achieve greater equity in health.

Table 2. Priority Recommendations for PNTP

Priority Recommendations
Sustain and optimize the Paediatric Nursing
Training Programme (PNTP).

Training Programme (PNTP).

Scale up paediatric nursing education across the country.

Develop strategies to increase awareness of the competencies of new PNTP graduates and enhance their professional recognition.

Develop expanded leadership opportunities for PNTP graduates and methods to sustain their expanded knowledge and competencies; thus strengthening their impact on clinical practice. Continue to monitor and evaluate how paediatric nurses are influencing organizational and clinical outcomes in their workplaces.

\section{Strategies}

Increase the number of nurses enrolled in the PNTP by (a) recruiting nurses from the districts, (b) targeting nurses in different roles (i.e., midwives and community nurses), and (c) developing mechanisms to sustain financial support for student education.

Address PNTP and institution-based resource challenges to ensure dedicated faculty and enhance training in clinical settings through collaborative planning with academic, GHS, and MOH partners.

Identify options for offering the PNTP curriculum beyond the current university and teaching hospital settings (e.g. colleges and district hospitals), and alternate delivery methods (e.g. workshops, webinars, e-modules).

Develop and further build on innovative teaching methodologies and provide opportunities for faculty development.

Clarify professional designation to be awarded following successful PNTP completion in association with partners within the Ghana health care system. Evaluate scope of practice, given new knowledge and skills obtained in the PNTP, in collaboration with the Ghana College of Nurses and Midwives, Nursing and Midwifery Council, Clinical Sites, School of Nursing, and graduate representatives.

Develop a sample job description based on PNTP competencies to guide institutions.

Develop opportunities for continuing education/ professional development for PNTP graduates to network (e.g. a formal Paediatric Nursing Association and informal communities of paediatric nursing practice in social media outlets). Develop educational outreach strategies (workshops) for preceptors and educators. Ensure that the monitoring and evaluation of paediatric nursing knowledge and skill is conducted over the short-, medium- and long- term (e.g. undertake descriptive case studies or comparative cohort studies to evaluate impact of the PNTP graduates over time). 


\section{Acknowledgments}

We are grateful to the PNTP students in Ghana for their enthusiasm and interest in child health. The initiative would not have been possible without the participation of the committee members and collaborators at the MOH, GHS, UG-SON, N\&MC, KBTH, Ghana Registered Nurses Association, and SickKids. We would also like to acknowledge the financial support for this research within the Ghana-SickKids PNTP by the Government of Canada provided through the Department of Foreign Affairs, Trade and Development as well as the SickKids Foundation and the Charles and Rita Field-Marsham Foundation. Jackie Boyce provided assistance with coding.

\section{Competing interests}

The authors declare that they have no competing interests.

\section{References}

[1] Millennium development goals indicators: The official United Nations site for the MDG indicators [Internet]. New York, NY: United Nations. 2000. Available from: http://mdgs.un.org/unsd/mdg/default.aspx.

[2] Save the Children UK. The child development index: holding governments to account for children's wellbeing [Internet]. London, UK: Save the Children; 2008. Available from:

http://www.savethechildren.org.uk/sites/default/files/docs/child-development-index.pdf.

[3] Alhassan RK, Spieker N, van Ostenberg P, Ogink A, Nketiah-Amponsah E, Rinke de Wit, TF. Association between health worker motivation and healthcare quality efforts in Ghana. Human Resources for Health [Internet]. 2013 Aug; 11(37): 1-11. Available from: http://www.human-resources-health.com/content/pdf/1478-4491-11-37.pdf.

[4] Snow RC, Asabir K, Mutumba M, Koomson E, Gyan K, Dzodzomenyo M, et al. Key factors leading to reduced recruitment and retention of health professionals in remote areas of Ghana: a qualitative study and proposed policy solutions. Human Resources for Health [Internet]. 2011May; 9(13): 1-11. Available from:

http://www.human-resources-health.com/content/pdf/1478-4491-9-13.pdf.

[5] United Nations Development Programme, Ghana; National Development Planning Commission, Government of Ghana. 2010 Ghana millenium development goals report [Internet]. Accra, GH: UNDP Ghana NDPC/GOC. 2012 Jul. Available from: http://www.gh.undp.org/content/dam/ghana/docs/Doc/Inclgro/UNDP_GH_IG_2010MDGreport_18102013.pdf.

[6] World Health Organization Department of Human Resources for Health. Increasing access to health workers in remote and rural areas through improved retention: global policy recommendations [Internet]. Geneva, CH: WHO Press; 2010. Available from: http://whqlibdoc.who.int/publications/2010/9789241564014_eng.pdf.

[7] Kwansah J, Dzodzomenyo M, Mutumba M, Asabir K, Koomson E, Gyakobo M, Agyei-Baffour P, Kruk ME, Snow RC. Policy talk: incentives for rural service among nurses in Ghana. Health Policy and Planning [Internet]. 2012 Feb; 27: 669-676. Available from: http://heapol.oxfordjournals.org/content/27/8/669.full.pdf+html. PMid:22349086 http://dx.doi.org/10.1093/heapol/czs016

[8] Donkor NT, Andrews LD. 21st century nursing practice in Ghana: challenges and opportunities. International Nursing Review. 2011; 58: 218-24. PMid:21554296 http://dx.doi.org/10.1111/j.1466-7657.2010.00856.x

[9] Sandelowski M. Focus on research methods: whatever happened to qualitative description? Research in Nursing \& Health. 2000; 23: 334-40. http://dx.doi.org/10.1002/1098-240X(200008)23:4<334::AID-NUR9>3.0.CO;2-G

[10] Ghana Statistical Service (GSS); Ghana Health Service (GHS); ICF Macro. Ghana demographic and health survey 2008 [Internet]. Accra, GH: GSS, GHS, and ICF Macro; 2009. Available from: http://dhsprogram.com/pubs/pdf/FR221/FR221[13Aug2012].pdf.

[11] Ritchie J, Spencer L, O’Connor W. Carrying out qualitative analysis. In: Ritchie J, Lewis J, editors. Qualitative research practice: a guide for social science students and researchers. Thousand Oaks, CA: Sage; 2003; 219-262.

[12] Frenk J, Chen L, Bhutta ZA, Cohen J, Crisp N, Evans T, et al. Health professionals for a new century: transforming education to strengthen health systems in an interdependent world. The Lancet. 2010; 376: 1923-58. http://dx.doi.org/10.1016/S0140-6736(10)61854-5

[13] Carrera LI, Tellez TE, D'Ottavio AE. Implementing a problem-based learning curriculum in an Argentinean medical school: implications for developing countries. Acad Med. 2003 Aug; 78(8): 798-801. PMid:12915370 http://dx.doi.org/10.1097/00001888-200308000-00010

[14] Murray JP, Wenger AFZ, Downes EA, Terrazas SB. Educating health professionals in low-resource countries: a global approach. New York: Springer Publishing Company; 2011. 
[15] van Berkel H, Scherpbier A, Hillen H, van der Vleuten C, editors. Lessons from problem-based learning. New York: Oxford University Press; 2010. http://dx.doi.org/10.1093/acprof:oso/9780199583447.001.0001

[16] Appiah-Denkyira E, Herbst CH, Soucat A, Lemiere C, Saleh K, editors. Toward interventions in human resources for health in Ghana: evidence for health workforce planning and results [Internet]. Washington, DC: The World Bank; 2012; 231. Available from: http://www.scribd.com/doc/130990887/Toward-Interventions-in-Human-Resources-for-Health-in-Ghana

[17] Ghana Health Workforce Observatory. Ghana human resources for health country profile, edition 2011 [Internet]. Ghana: Ghana Health Workforce Observatory; 2010 Feb. Available from: http://www.hrh-observatory.afro.who.int/images/Document_Centre/ghana_hrh_country_profile.pdf.

[18] Aseno SB. Financing health education in Ghana. The Ghanain Chronicle [Internet]. 2013 Sept 13. Available from: http://thechronicle.com.gh/financing-health-education-in-ghana-policy-alternatives/.

[19] Global Health Workforce Alliance; World Health Organization. A universal truth: no health without a workforce [Internet]. Geneva, CH: WHO Press; 2013. Available from: http://www.who.int/workforcealliance/knowledge/resources/GHWA_AUniversalTruthReport.pdf.

[20] Smithers N (World Bank Institute Capacity Development and Results). The importance of stakeholder ownership for capacity development results [Internet]. Washington, DC: The World Bank; 2011 May [cited 2014 Sept 4]. 48 p. Available from: https://wbi.worldbank.org/wbi/Data/wbi/wbicms/files/drupal-acquia/wbi/The_Importance.pdf.

[21] Kolars JC, Cahill K, Donkor P, Kaaya E, Lawson A, Serwadda D, Sewankambo N. Perspective: partnering for medical education in Sub-Saharan Africa: seeking the evidence for effective collaborations. Academic Medicine. 2012 Feb; 87(2): $216-20$. PMid:22189887 http://dx.doi.org/10.1097/ACM.0b013e31823ede39 